J. Korean Math. Soc. 52 (2015), No. 3, pp. 469-487

http://dx.doi.org/10.4134/JKMS.2015.52.3.469

\title{
HYBRID ON-OFF CONTROLS FOR AN HIV MODEL BASED ON A LINEAR CONTROL PROBLEM
}

\author{
Tae Soo Jang, Jungeun Kim, Hee-Dae Kwon, and Jeehyun Lee
}

\begin{abstract}
We consider a model of HIV infection with various compartments, including target cells, infected cells, viral loads and immune effector cells, to describe HIV type 1 infection. We show that the proposed model has one uninfected steady state and several infected steady states and investigate their local stability by using a Jacobian matrix method. We obtain equations for adjoint variables and characterize an optimal control by applying Pontryagin's Maximum Principle in a linear control problem. In addition, we apply techniques and ideas from linear optimal control theory in conjunction with a direct search approach to derive on-off HIV therapy strategies. The results of numerical simulations indicate that hybrid on-off therapy protocols can move the model system to a "healthy" steady state in which the immune response is dominant in controlling HIV after the discontinuation of the therapy.
\end{abstract}

\section{Introduction}

HIV is a type of lentivirus that infects core cells in the immune system, including CD4+ T cells and macrophages, and then causes Acquired Immune Deficiency Syndrome (AIDS). Recent years have witnessed considerable advances in the treatment of HIV patients. More than 20 FDA-approved anti-HIV drugs are currently available. Two major types of anti-HIV drugs are reverse transcriptase inhibitors (RTIs) and protease inhibitors (PIs). An efficient treatment regimen typically used for suppressing the replication of HIV is HAART (highly active antiretroviral therapy), which combines at least three anti-HIV drugs. Although the use of HAART has led to sharp declines in HIV-related morbidity and mortality, current treatment methods cannot clear HIV in patients, and therefore HIV patients must continue HAART for life [19, 21, 26].

Lifelong HAART can give rise to side effects, toxicity, adherence, drug resistance, and high costs $[8,13]$. These risks and problems strongly motivate the consideration of treatment strategies known as structured treatment interruptions (STIs) or on-off therapy strategies. These treatment strategies have

Received April 29, 2014; Revised October 29, 2014.

2010 Mathematics Subject Classification. 49J15, 92C50, 93C15.

Key words and phrases. HIV dynamics, linear optimal control, STI, bang-bang control. 
received increasing attention in recent years, but their efficacy and safety have been controversial. Many studies have considered specific STI strategies involving different interruption intervals and decision rules for stopping and initiating therapy [17, 20, 23, 28]. Tang et al. [28] use a piecewise model of HIV dynamics to explore STI strategies guided by CD4+ T cell counts. Palacios et al. [20] examine the viral, immune, and clinical impacts of a STI program in three cycles of 12 weeks on and 4 weeks off on children infected with HIV.

There are some evidences that the HIV specific cytotoxic T lymphocyte response (CD8+ immune effector cells) activity increases in patients experiencing a viral rebound [20,27]. Therefore, a number of studies have developed methods that can boost immune effector cells through vaccine or treatment interruptions once patients have suppressed the viral replication [30]. During interruptions, the viral load typically increases, thereby stimulating or reactivating CD 8 immune effector cells. The repeated discontinuation of antiretroviral therapy can enable patients to maintain immune control of HIV with no treatment [16].

Many studies have explored the benefits of STIs, but they have considered a wide range of interruption schedules and thus produced mixed results. Therefore, there is no general consensus on optimal STI schedules. A good way to derive optimal STI strategies is to use a mathematical model of HIV infection in conjunction with control theory. A number of studies have explored optimal control of HIV infection by using different models and objective functionals to suggest continuous optimal treatment strategies $[1,2,10,14,29]$. In addition, many studies have considered feedback control of HIV infection [3, 4, 6, 25]. Banks et al. [4] examine optimal feedback control of HIV infection by taking a state-dependent Riccati equation (SDRE) approach and explore the problem of designing a state estimator for use in nonlinear feedback control laws because only partial measurements of the state are available in many practical problems.

Adams et al. [1, 2] investigate optimal control of viremia by considering a number of drug-structured treatment interruptions and propose a direct search approach using ideas from dynamic programming to obtain suboptimal on-off treatment strategies, but because this approach is limited in that it can lead to a large number of cost functional evaluations, they use five-day segments and a subperiod method to reduce the number of iterations. In the present paper, we investigate the problem of linear optimal control by using a mathematical model of HIV infection consisting of a system of ordinary differential equations (ODEs). We then apply the conjugate gradient algorithm to the linear control problem in conjunction with the direct search approach to derive STI strategies. The numerical simulations demonstrate that the derived on-off therapy protocols can move the model system to a "healthy" steady state in which the immune response is dominant in more efficiently controlling HIV after the discontinuation of the therapy.

The rest of this paper is organized as follows: Section 2 describes and analyzes this paper's mathematical model. Section 3 formulates the problem of 
linear optimal control, derives the corresponding optimality system, and characterizes the optimal control function. Section 4 introduces an hybrid algorithm combining the conjugate gradient method and the direct search approach to derive on-off therapy strategies. The numerical results demonstrate the effectiveness of these STI schedules. Section 5 provides concluding remarks.

\section{HIV model}

This section introduces an HIV model involving an immune response described through Michaelis-Menten-type saturation nonlinearity adapted from Adams et al. [1, 2]. A system of ODEs describing compartmental infection dynamics can be given by

$$
\begin{array}{ll}
\text { Target: } & \dot{S}=\lambda-d S-\left(1-\alpha_{1} \varepsilon\right) k V S \\
\text { Infected: } & \dot{I}=\left(1-\alpha_{1} \varepsilon\right) k V S-\delta I-m E I \\
\text { Virus: } & \dot{V}=\left(1-\alpha_{2} \varepsilon\right) N_{T} \delta I-c V \\
\text { Immune effectors: } & \dot{E}=\lambda_{E}+\frac{b_{E} I}{I+K_{b}} E-\frac{d_{E} I}{I+K_{d}} E-\delta_{E} E
\end{array}
$$

together with an initial condition

$$
[S(0), I(0), V(0), E(0)]
$$

TABLE 1. Values of parameters of model (2.1).

\begin{tabular}{ccc|ccc} 
parameter & value & units & parameter & value & units \\
\hline$\lambda$ & 10.0 & $\frac{\text { cells }}{\mathrm{mm}^{3} \cdot d a y}$ & $N_{T}$ & 100 & $\frac{\text { virions }}{\text { cell }}$ \\
$d$ & 0.01 & $\frac{1}{d a y}$ & $c$ & 13 & $\frac{1}{d a y}$ \\
$\alpha_{1}$ & 0.7 & - & $\lambda_{E}$ & $10^{-3}$ & $\frac{\text { cells }}{\mathrm{mm}^{3} \cdot d a y}$ \\
$\varepsilon$ & $\in[0,1]$ & - & $b_{E}$ & 0.3 & $\frac{1}{d a y}$ \\
$k$ & $8.0 \times 10^{-4}$ & $\frac{\mathrm{mm}^{3}}{\text { virions.day }^{3}}$ & $K_{b}$ & 0.1 & $\frac{\text { cells }}{m^{3}}$ \\
$\delta$ & 0.7 & $\frac{1}{d a y}$ & $d_{E}$ & 0.25 & $\frac{1}{d a y}$ \\
$m$ & $1.0 \times 10^{-2}$ & $\frac{m m^{3}}{\text { cells } \cdot d a y}$ & $K_{d}$ & 0.5 & $\frac{\text { cells }}{m m^{3}}$ \\
$\alpha_{2}$ & 0.3 & - & $\delta_{E}$ & 0.1 & $\frac{1}{d a y}$ \\
\hline
\end{tabular}

This model includes four key compartments: uninfected target cells, CD4+ T cells $\left(S\right.$, cells $\left./ \mathrm{mm}^{3}\right)$, infected target cells $\left(I\right.$, cells $\left./ \mathrm{mm}^{3}\right)$, free viruses $(V$, copies $/ \mathrm{mm}^{3}$ ), and immune response, cytotoxic T lymphocytes (CTLs) (E, cells $/ \mathrm{mm}^{3}$ ). Here we focus on those interactions relevant to drug treatment and STI scenarios and discuss the model in the context of its representation of two methods for controlling HIV infection: RTIs and PIs.

The terms involving $k S V$ represent the infection process in which infected cells $I$ result from encounters between uninfected target cells $S$ and free virus $V$. In the infectivity terms, drug efficacy $\alpha_{1} \varepsilon(t)$ models an RT inhibitor that blocks new infections by preventing the conversion of HIV RNA into DNA, 
which is a crucial part of the viral life cycle. Therefore, the virus infection rate $k$ is reduced by a factor of $\left(1-\alpha_{1} \varepsilon\right)$. On the other hand, PIs do not directly inhibit the infectiousness of the virus. Instead, they alter some part of the viral assembly process during the final stage of the viral life cycle and thus cause the production of defective and noninfectious viruses. Drug efficacy $\alpha_{2} \varepsilon(t)$ represents the fraction of noninfectious viruses produced from PI activity, and therefore productivity $N_{T}$ decreases to $\left(1-\alpha_{2} \varepsilon(t)\right) N_{T}$. We consider the control term $0 \leq \varepsilon(t) \leq 1$, and therefore $\varepsilon(t)=0$ and $\varepsilon(t)=1$ represent no- and fulltreatment scenarios, respectively.

Finally, infected cells $I$ may be cleared through the action of immune effector cells (CTLs), denoted by E. Bonhoeffer et al. [5] suggest the dynamics of the immune response. The joint presence of infected cells and existing immune effector cells stimulates the proliferation of additional immune effector cells. In addition, the third term in the equation for immune effector cells $E$ represents the immune impairment at high viral load. CTLs detect and lyse infected cells, thereby killing them. Their action is represented by the term $m E I$. That is, infected cells die at the rate $m E$, which is dependent on the density of immune effector cells. The inclusion of immune effector cells reflects the belief that they play a crucial role in the context of STIs. The treatment strategies that can boost these cells to the point of immune control are discussed later.

\subsection{Analysis of the HIV model}

The mathematical model (2.1) contains numerous parameters that must be assigned before any numerical simulations. In specifying model parameters, we maximize the use of values similar to those reported or justified in previous researches. Table 1 summarizes the numerical values for these parameters, which are extracted mainly from Bonhoeffer et al. [5] and Callaway and Perelson $[7]$.

We let $x=(S, I, V, E)^{T}$ denote the vector of model states and represent model (2.1) as

$$
\frac{d x(t)}{d t}=f(t, x)
$$

where $f(t, x)$ is the right side of the ODE system. We use Maple to solve $f(t, x)=0$ for finding the steady state $\bar{x}_{k}$ with the parameter values in Table 1. Then we calculate the Jacobian matrix

$$
\frac{\partial f(t, x)}{\partial x}=\left[\frac{\partial f_{i}(t, x)}{\partial x_{j}}\right]
$$

of the ODE system to consider the stability of the steady state. Because we are interested in this stability for off-treatment steady state values, we set $\varepsilon=0$. 
The Jacobian matrix is

$$
\left(\begin{array}{cccc}
-d-k V & 0 & -k S & 0 \\
k V & -\delta-m E & k S & -m I \\
0 & N_{T} \delta & -c & 0 \\
0 & A_{4,2} E & 0 & A_{4,4} I-\delta_{E}
\end{array}\right)
$$

where

$A_{4,2}=\frac{b_{E} K_{b}}{\left(I+K_{b}\right)^{2}}-\frac{d_{E} K_{d}}{\left(I+K_{d}\right)^{2}} \quad$ and $\quad A_{4,4}=\frac{b_{E}}{I+K_{b}}-\frac{d_{E}}{I+K_{d}}$.

TABLE 2. Off-treatment $(\varepsilon \equiv 0)$ steady states $\left(\right.$ cells $\left./ \mathrm{mm}^{3}\right)$ for model (2.1) (non-physical steady states are omitted).

\begin{tabular}{|c|c|c|c|c|}
\hline & $E Q_{0}$ & $E Q_{1}$ & $E Q_{2}$ & $E Q_{3}$ \\
\hline $\mathrm{S}$ & 1,000 & 162.571 & 580.770 & 968.863 \\
$\mathrm{I}$ & 0 & 11.958 & 1.675 & 0.075 \\
$\mathrm{~V}$ & 0 & 64.389 & 9.023 & 0.402 \\
$\mathrm{E}$ & 0.01 & 0.031 & 180.178 & 347.356 \\
\hline local stability & unstable & stable & unstable & stable \\
\hline
\end{tabular}

Based on general ODE theory, it is guaranteed that if all eigenvalues of the Jacobian matrix have negative real parts, then the equilibrium $\bar{x}_{k}$ is locally asymptotically stable. Given the specified parameters under no treatment $(\varepsilon=$ 0 ), the model has several steady states, as shown in Table 2. Here we have a locally unstable virus-free equilibrium $E Q_{0}$ such that

$$
S=1000, \quad I=0, \quad V=0, \quad E=0.01,
$$

which represents an HIV-free individual, as well as two locally stable equilibria for an HIV patient with no treatment. These stable steady states are as follows:

$$
\begin{array}{cccc}
\text { "unhealthy" }\left(E Q_{1}\right): S=162.571, & I=11.958, & V=64.389, & E=0.031 ; \\
\text { "healthy" }\left(E Q_{3}\right): S=968.863, & I=0.075, & V=0.402, & E=347.356 .
\end{array}
$$

Here the "unhealthy" steady state corresponds to a dangerously high viral set point, depleted $\mathrm{T}$ cells, and the minimal immune response, whereas the "healthy" steady state represents immune control of viral infection and the restoration of CD4+ T cells.

In general, the introduction of a low viral load causes the model system converge to the "unhealthy" steady state $E Q_{1}$ under no treatment. Here we derive on-off control functions (STI schemes) which move the model system from the virus-dominant equilibrium $E Q_{1}$ to the immune-dominant equilibrium $E Q_{3}$ (see Figure 1). 


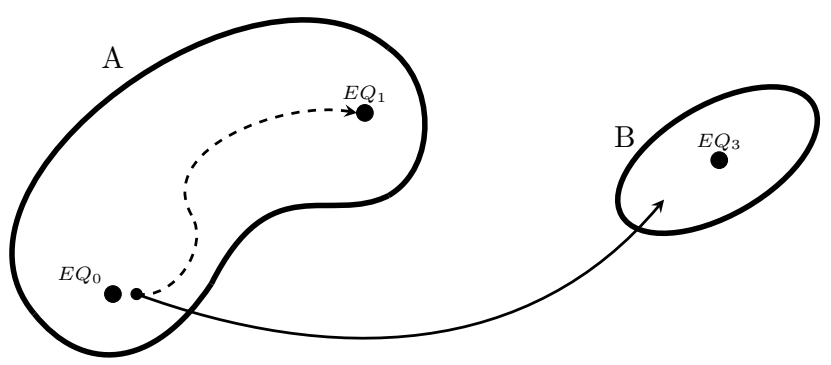

FiguRE 1. $E Q_{0}$ : uninfected locally unstable virus-free equilibrium; $E Q_{1}$ : "unhealthy" locally asymptotically stable equilibrium with the attraction region $A ; E Q_{3}$ : "healthy" locally asymptotically stable equilibrium with the attraction region $B$; dashed line (--): uncontrolled trajectory; and solid line $(-)$ : controlled trajectory.

\section{Linear optimal control problem}

Previous studies have employed optimal control techniques to investigate dynamic drug therapies for HIV patients. Such studies have used various types of mathematical models and/or objective functionals for HIV dynamics. Here we consider practical therapeutic options in a clinical setting that can minimize the total viral load and boost the immune response while allowing for STIs. To design an on-off HIV therapeutic strategy, we first formulate a linear optimal control problem. In addition to the mathematical model described in equation (2.1) for HIV dynamics, we consider a control problem with the objective functional given by

$$
J(\varepsilon)=\int_{0}^{t_{f}}[P \varepsilon(t)+Q V(t)-R E(t)] d t,
$$

where $\varepsilon(t)$ is the control variable representing time-dependent treatment. The parameters $P, Q$, and $R$ are weight constants for the control input, virus, and immune effector, respectively.

The first term in (3.1) represents the systemic cost of the drug treatment. The objective functional (3.1) expresses this paper's goal to minimize both the HIV population and the systemic cost to the body while maximizing the immune response. Therefore, we seek the optimal control $\varepsilon^{*}$ such that

$$
J\left(\varepsilon^{*}\right)=\min \{J(\varepsilon) \mid \varepsilon \in \mathfrak{U}\}
$$

subject to the system of ODEs (2.1), where $\mathfrak{U}=\{\varepsilon \mid \varepsilon$ is piecewise continuous, $\varepsilon(t) \in U=[0,1]$, and $\left.t \in\left[0, t_{f}\right]\right\}$ is the control set. 


\subsection{Existence of optimal controls}

The existence of a solution to the optimal control problem can be obtained by verifying sufficient conditions. We refer to the conditions in Theorem III.4.1 and its corresponding Corollary in [11].

Theorem 3.1. There exists an optimal control $\varepsilon^{*}$ to the linear optimal control problem (3.2).

Proof. Let $f(t, x, \varepsilon)$ be the right-hand side of (2.1). The boundedness of solutions to the system (2.1) for a finite time interval is needed to establish the conditions. Note that the quantities $S, I, V$, and $E$ decrease only in proportion to their present sizes, and therefore all variables remain nonnegative if their initial values are nonnegative. To establish upper bounds on solutions, we consider the supersolutions $\check{S}, \check{I}, \check{V}$, and $\check{E}$ satisfying

$$
\frac{d \check{S}}{d t}=\lambda, \quad \frac{d \check{I}}{d t}=C k \check{V}, \quad \frac{d \check{V}}{d t}=N_{T} \delta \check{I}, \quad \text { and } \quad \frac{d \check{E}}{d t}=\lambda_{E}+b_{E} \check{E} .
$$

Then $\check{S}$ and $\check{E}$ are uniformly bounded in the finite time interval, and so are $\check{I}$ and $\check{V}$ since they satisfy the linear system with bounded coefficients. Therefore, there exists a constant $C$ such that

$$
|f(t, 0,0)| \leq C, \quad\left|f_{x}(t, x, \varepsilon)\right| \leq C(1+|\varepsilon|), \quad \text { and } \quad\left|f_{\varepsilon}(t, x, \varepsilon)\right| \leq C .
$$

By (3.3), we know that $f$ is continuous. In addition, there exist positive constants $C_{1}$ and $C_{2}$ such that $|f(t, x, u)| \leq C_{1}(1+|x|+|u|)$ and $\mid f\left(t, x_{1}, u\right)-$ $f\left(t, x_{2}, u\right)\left|\leq C_{2}\right| x_{1}-x_{2} \mid(1+|u|)$. Therefore there exists a unique solution to (2.1) for a constant control, which implies that the admissible set of all solutions to system (2.1) with the corresponding control in $\mathfrak{U}$ is non-empty [18, Theorem 9.2.1].

The function $f(t, x, \varepsilon)$ can be expressed in the generic form

$$
f(t, x, \varepsilon)=F(x(t))+G(x(t)) \varepsilon(t),
$$

where

$$
F(x)=\left(\begin{array}{c}
\lambda-d S-k V S \\
k V S-\delta I-m E I \\
N_{T} \delta I-c V \\
\lambda_{E}+\frac{b_{E} I E}{I+K_{b}}-\frac{d_{E} I E}{I+K_{d}}-\delta_{E} E
\end{array}\right) \quad \text { and } \quad G(x)=\left(\begin{array}{c}
\alpha_{1} k V S \\
-\alpha_{1} k V S \\
-\alpha_{2} N_{T} \delta I 0
\end{array}\right)
$$

Therefore, $f$ is linear in the control $\varepsilon$ with the coefficients dependent on time $t$ and the state variable $x$.

The set $U=[0,1]$ is compact and convex, and by definition, the integrand of the cost functional is convex in $U$. Having verified the conditions, we apply Theorem III.4.1 and Corollary III.4.2 in [11] to conclude that there exists an optimal control function. 


\subsection{Optimality system}

We now characterize an optimal control function by using Pontryagin's Maximum Principle [22]. We convert the constrained minimization problem (3.2) into an unconstrained one to obtain an optimality system, which is a necessary condition for an optimal solution.

Using the notation (3.4), we can write the HIV model (2.1) as

$$
\dot{x}=F(x)+G(x) \varepsilon,
$$

where $F(x)$ and $G(x)$ are described in (3.5). Let $F_{i}$ and $G_{i}(i=1,2,3,4)$ denote components of $F$ and $G$, respectively:

$$
F=\left(F_{1}, F_{2}, F_{3}, F_{4}\right)^{T} \text { and } \quad G=\left(G_{1}, G_{2}, G_{3}, G_{4}\right)^{T} .
$$

Note that

$$
\dot{F}=\frac{\partial F}{\partial x} \dot{x} \quad \text { and } \quad \dot{G}=\frac{\partial G}{\partial x} \dot{x} .
$$

Theorem 3.2. Given the optimal control $\varepsilon^{*}$ and solutions to the corresponding state system (2.1), there exist adjoint variables $\xi=\left(\xi_{1}, \xi_{2}, \xi_{3}, \xi_{4}\right)^{T}$ satisfying

$$
\dot{\xi}=-\left([A(x)]^{T}+[B(x)]^{T} \varepsilon\right) \xi-C \quad \text { and } \quad \xi\left(t_{f}\right)=0,
$$

where

$$
A(x)=\frac{\partial F}{\partial x}, \quad B(x)=\frac{\partial G}{\partial x}, \quad \text { and } \quad C=(0,0, Q,-R)^{T} .
$$

Further, if we define the switching function $\psi$ by

$$
\psi(t):=P+\xi^{T} G(x(t)),
$$

then $\varepsilon^{*}$ can be expressed as

$$
\varepsilon^{*}(t)=\left\{\begin{array}{ccc}
0 & \text { if } & \psi>0 \\
-\frac{\mathcal{P}(x, \xi)}{\mathcal{Q}(x, \xi)} & \text { if } & \psi=0 \\
1 & \text { if } & \psi<0
\end{array}\right.
$$

where

$$
\begin{aligned}
& \mathcal{P}(x, \xi)=\xi^{T}\left[\left(A^{2}+C^{T} A-\widetilde{A}_{1}\right) G+\left(B A-2 A B+\widetilde{B}_{1}\right) F\right]+C^{T}(A G-2 B F), \\
& \mathcal{Q}(x, \xi)=\xi^{T}\left[\left(2 B A-\widetilde{A}_{2}-A B\right) G+\left(\widetilde{B}_{2}-B^{2}\right) F\right]-C^{T} B G
\end{aligned}
$$

where $\widetilde{A}_{1}, \widetilde{A}_{2}, \widetilde{B}_{1}$, and $\widetilde{B}_{2}$ are defined in the proof.

Proof. The Hamiltonian equation is given by

$$
H=P \varepsilon+Q V-R E+\xi^{T} \dot{x}=P \varepsilon+Q V-R E+\xi^{T}(F(x)+G(x) \varepsilon) .
$$


Taking the first variations with respect to $S, I, V$, and $E$ yields the adjoint, or costate, equations

$$
\begin{aligned}
& \dot{\xi_{1}}=-\frac{\partial H}{\partial S}=-\xi^{T} \frac{\partial \dot{x}}{\partial S}, \\
& \dot{\xi_{2}}=-\frac{\partial H}{\partial I}=-\xi^{T} \frac{\partial \dot{x}}{\partial I} \\
& \dot{\xi_{3}}=-\frac{\partial H}{\partial V}=-\xi^{T} \frac{\partial \dot{x}}{\partial V}-Q, \\
& \dot{\xi_{4}}=-\frac{\partial H}{\partial E}=-\xi^{T} \frac{\partial \dot{x}}{\partial E}+R,
\end{aligned}
$$

with the terminal condition $\xi\left(t_{f}\right)=0$.

By the simple calculations of $\frac{\partial \dot{x}}{\partial S}, \frac{\partial \dot{x}}{\partial I}, \frac{\partial \dot{x}}{\partial V}$, and $\frac{\partial \dot{x}}{\partial E}$, we can write the system of adjoint equations as

$$
\dot{\xi}=-\left([A(x)]^{T}+[B(x)]^{T} \varepsilon\right) \xi-C,
$$

where

$$
\begin{aligned}
& A(x):=\frac{\partial F}{\partial x}=\left(\begin{array}{cccc}
-d-k V & 0 & -k S & 0 \\
k V & -\delta-m E & k S & -m I \\
0 & N_{T} \delta & -c & 0 \\
0 & A_{4,2} E & 0 & A_{4,4} I-\delta_{E}
\end{array}\right) \\
& A_{4,2}=\frac{b_{E} K_{b}}{\left(I+K_{b}\right)^{2}}-\frac{d_{E} K_{d}}{\left(I+K_{d}\right)^{2}} \quad \text { and } \quad A_{4,4}=\frac{b_{E}}{I+K_{b}}-\frac{d_{E}}{I+K_{d}},
\end{aligned}
$$

and

$$
B(x):=\frac{\partial G}{\partial x}=\left(\begin{array}{cccc}
\alpha_{1} k V & 0 & \alpha_{1} k S & 0 \\
-\alpha_{1} k V & 0 & -\alpha_{1} k S & 0 \\
0 & -\alpha_{2} N_{T} \delta & 0 & 0 \\
0 & 0 & 0 & 0
\end{array}\right)
$$

The equation $\frac{\partial H}{\partial \varepsilon}=0$ contains no information on the control. Therefore, to characterize the optimal control, we define the switching function

$$
\psi:=\frac{\partial H}{\partial \varepsilon}=P+\xi^{T} G(x) .
$$

Then the optimal control is given by

$$
\varepsilon^{*}=\left\{\begin{array}{ccc}
0 & \text { if } & \psi>0 \\
\text { singular } & \text { if } & \psi=0 \\
1 & \text { if } & \psi<0
\end{array}\right.
$$

If $\psi=0$ cannot be sustained over an interval but occurs only at finitely many points, then we have a bang-bang control. In this case, $\varepsilon^{*}$ is a piecewise constant function switching between only upper and lower bounds. If $\psi \equiv 0$ over an interval, then $\varepsilon^{*}$ is singular for that interval. Here we must find the characterization of $\varepsilon^{*}$ for this interval, called singular arc, by using other information. 
To address the issue of the singular arcs, we assume that the switching function $\psi$ is zero over the interval $\left(t_{1}, t_{2}\right)$. Setting the first derivative of the switching function to zero, we obtain

$$
\begin{aligned}
0=\dot{\psi} & =\dot{\xi}^{T} G+\xi^{T} \dot{G} \\
& =-\left(A^{T} \xi+B^{T} \xi \varepsilon+C\right)^{T} G+\xi^{T}(B F+B G \varepsilon) \\
& =-\xi^{T} A G-C^{T} G+\xi^{T} B F .
\end{aligned}
$$

Since equation (3.10) has no information on the control $\varepsilon$, we further investigate the second derivative of the switching function. By (3.6) and (3.9), we have

$$
\begin{aligned}
0=\ddot{\psi}=- & \dot{\xi}^{T} A G-\xi^{T} \dot{A} G-\xi^{T} A \dot{G}-C^{T} \dot{G}+\dot{\xi}^{T} B F+\xi^{T} \dot{B} F+\xi^{T} B \dot{F} \\
=( & \left.A^{T} \xi+B^{T} \xi \varepsilon+C\right)^{T} A G-\xi^{T} \dot{A} G-\xi^{T} A(B F+B G \varepsilon) \\
& -C^{T}(B F+B G \varepsilon)-\left(A^{T} \xi+B^{T} \xi \varepsilon+C\right)^{T} B F \\
& +\xi^{T} \dot{B} F+\xi^{T} B(A F+A G \varepsilon) .
\end{aligned}
$$

We then compute and express the derivatives of $A$ and $B$ as follows:

$$
\dot{A}=\widetilde{A}_{1}(x)+\widetilde{A}_{2}(x) \varepsilon,
$$

where

$$
\begin{gathered}
\widetilde{A}_{1}(x)=\left(\begin{array}{cccc}
-k F_{3} & 0 & -k F_{1} & 0 \\
k F_{3} & -m F_{4} & k F_{1} & -m F_{2} \\
0 & 0 & 0 & 0 \\
0 & A_{4,2} F_{4}+\widetilde{A}_{0} E F_{2} & 0 & A_{4,4} F_{2}
\end{array}\right), \\
\widetilde{A}_{2}(x)=\left(\begin{array}{cccc}
-k G_{3} & 0 & -k G_{1} & 0 \\
k G_{3} & -m G_{4} & k G_{1} & -m G_{2} \\
0 & 0 & 0 & 0 \\
0 & \widetilde{A}_{0} E G_{2} & 0 & A_{4,4} G_{2}
\end{array}\right),
\end{gathered}
$$

and

Similarly,

$$
\widetilde{A}_{0}=2\left(\frac{d_{E} K_{d}}{\left(I+K_{d}\right)^{3}}-\frac{b_{E} K_{b}}{\left(I+K_{b}\right)^{3}}\right)
$$

where

$$
\dot{B}=\widetilde{B}_{1}(x)+\widetilde{B}_{2}(x) \varepsilon,
$$

$$
\widetilde{B}_{1}(x)=\left(\begin{array}{cccc}
\alpha_{1} k F_{3} & 0 & \alpha_{1} k F_{1} & 0 \\
-\alpha_{1} k F_{3} & 0 & -\alpha_{1} k F_{1} & 0 \\
0 & 0 & 0 & 0 \\
0 & 0 & 0 & 0
\end{array}\right)
$$

and

$$
\widetilde{B}_{2}(x)=\left(\begin{array}{cccc}
\alpha_{1} k G_{3} & 0 & \alpha_{1} k G_{1} & 0 \\
-\alpha_{1} k G_{3} & 0 & -\alpha_{1} k G_{1} & 0 \\
0 & 0 & 0 & 0 \\
0 & 0 & 0 & 0
\end{array}\right)
$$


By substituting $\dot{A}$ and $\dot{B}$ into (3.11), we characterize the optimal control:

$$
\varepsilon=-\frac{\mathcal{P}(x, \xi)}{\mathcal{Q}(x, \xi)}
$$

where

$$
\mathcal{P}(x, \xi)=\xi^{T}\left[\left(A^{2}+C^{T} A-\widetilde{A}_{1}\right) G+\left(B A-2 A B+\widetilde{B}_{1}\right) F\right]+C^{T}(A G-2 B F)
$$

and

$$
\mathcal{Q}(x, \xi)=\xi^{T}\left[\left(2 B A-\widetilde{A}_{2}-A B\right) G+\left(\widetilde{B}_{2}-B^{2}\right) F\right]-C^{T} B G .
$$

We note that the singular control is admissible only if the value lies in the interval $[0,1]$ and the generalized Legendre-Clebsch condition is satisfied, that is,

$$
\frac{\partial}{\partial \varepsilon}\left[\frac{d^{2}\left(\frac{\partial H}{\partial \varepsilon}\right)}{d t^{2}}\right]=\mathcal{Q}(x, \xi)<0 .
$$

\section{Hybrid on-off controls}

We now determine an optimal solution to problem (3.2) by solving the optimality system. We have bang-bang controls for some intervals with a nonzero switching function and obtain continuous singular controls for some intervals in which the switching function is zero. However, a continuous therapy is not practical because in a clinical setting, treatment can only be altered at certain intervals. In this section, we derive on-off HIV therapy strategies for the whole interval that can provide clinical benefits similar to those of continuous treatment while allowing for STIs. For this, we approximate the continuous singular control through on-off control functions by taking the direct search approach.

In the direct search approach, we assume that the time-discretized control $\varepsilon$ is a vector consisting of only 0 or 1 . If a component of the control vector is 0 , then there is no drug treatment that day, whereas if it is 1 , then there is full drug treatment. The size of the control vector is as large as the number of treatment days. For example, if we consider a drug treatment strategy over $n$ days, then the size of the control vector is $1 \times n$. A set of all such control vectors is denoted by $\Lambda$. The objective is to identify the optimal control $\varepsilon^{*}$ satisfying

$$
J\left(\varepsilon^{*}\right)=\min _{\varepsilon \in \Lambda} J(\varepsilon)
$$

subject to the state system (2.1), where $J(\varepsilon)$ is defined by (3.1).

Since the number of elements of set $\Lambda$ is finite, the existence of an optimal control vector is guaranteed. In this search method, we can begin by selecting any vector $\varepsilon$ from set $\Lambda$ and then solving the state system with a control function representing the interpolation of the vector $\varepsilon$. We then select another $\varepsilon$ from set $\Lambda$ and again solve the state system in the same way. By comparing the values of the objective functional $J$, we select the control vector corresponding to the lower value of the cost functional. By iterating this strategy over all 
possible $\varepsilon$ from set $\Lambda$, we obtain the optimal control vector $\varepsilon^{*}$. However, this strategy to obtain the optimal control vector can lead to a large number of cost functional evaluations and thus a large number of solutions to the state system (2.1).

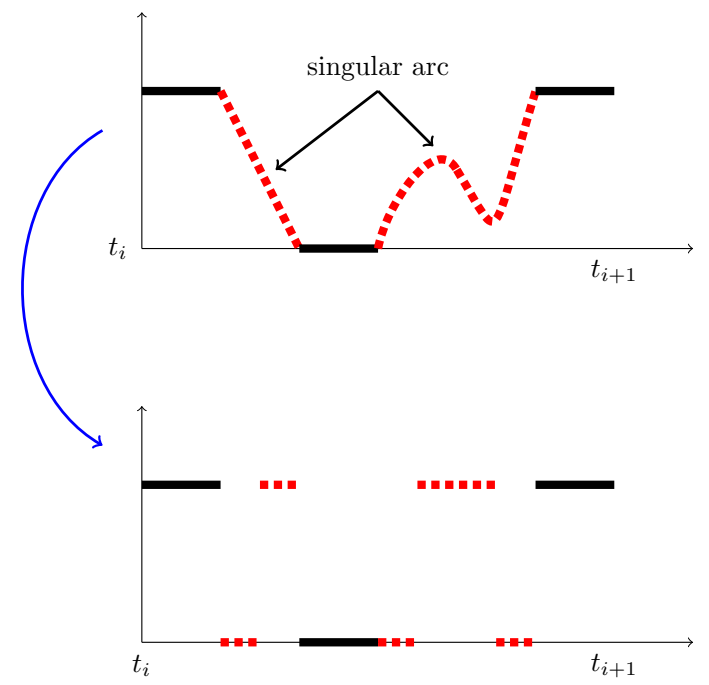

FiguRE 2. The linear optimal control (upper graph) and the hybrid on-off control (lower graph).

In the example, the number of cost functional evaluations is $2^{990}$ because the duration of treatment periods is assumed to be 990 days, which makes this approach computationally infeasible. Previous studies have suggested two approaches to reduce the number of iterations $[2,1]$. One approach is to use segments of several days instead of one-day segments. This is not only useful for reducing the computational burden but also more practical because it is not clinically feasible for drug schedules to allow for daily changes. Based on three-day segments, the size of each control vector decreases to $1 \times 330$ from $1 \times 990$. Therefore, the number of iterations decreases to $2^{330}$, which is still quite large.

The second way to address this problem is to consider subperiods such as $[0,30],[0,60],[0,90], \ldots,[0,990]$. In this method, we preferentially find an optimal STI control $\varepsilon_{1}^{*}$ over the first subperiod $[0,30]$ by using the aforementioned technique for reducing iterations (i.e., three-day segments). Since the size of $\varepsilon_{1}^{*}$ is $1 \times 10$ for three-day segments over 30 days, the number of iterations to obtain optimal solutions is $2^{10}=1024$. In the second step, we consider the following control vector for the subperiod $[0,60]$ :

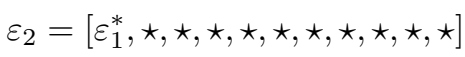


where $\star$ is 0 or 1 . That is, we fix the optimal STI $\varepsilon_{1}^{*}$ as the first 10 elements of the control $\varepsilon_{2}$ and iterate $\varepsilon_{2}$ to find the last 10 elements of the control that produces an optimal STI control $\varepsilon_{2}^{*}$ for the subperiod $[0,60]$. In this case, the number of iterations is also just $2^{10}=1024$, and therefore we can obtain it quickly. We repeat this process to find an optimal STI control $\varepsilon_{3}^{*}$ for $[0,90], \varepsilon_{4}^{*}$ for $[0,120]$, and so on. The STI control obtained for the whole period $[0,990]$ is $\varepsilon^{*}=\varepsilon_{33}^{*}$. We refer to this STI control driven by the aforementioned direct search approach as the "simple STI". Here it should be emphasized that the simple STI control $\varepsilon^{*}$ is suboptimal.

We now consider a hybrid on-off control that combines the linear optimal control and the direct search method. We first use an iteration method of the conjugate gradient type to solve the linear optimal control problem in each subperiod. The conjugate gradient algorithm is a powerful scheme for solving large-scale minimization problems $[9,12,15,24]$. Using an initial guess for the control variable, we solve the state system (2.1) with initial conditions forward in time and then solve the adjoint system (3.7) with terminal conditions backward in time. We update the control in each iteration by using the conjugate gradient algorithm. Using the algorithm, we obtain a control that is continuous on the singular arc and bang-bang otherwise in each subperiod (see the upper graph in Figure 2). To derive an on-off control for the subperiod, we apply the direct search method to the singular part.

We first simulate early infection by introducing very low levels of the virus particle and the infected CD4 $+\mathrm{T}$ cell density as follows:

$$
S(0)=10^{3}, \quad I(0)=10^{-7}, \quad V(0)=10^{-3} \text { and } E(0)=10^{-2} .
$$

We choose the weight constants $P=1, Q=10^{-2}$, and $R=5 \times 10^{2}$ to balance the differences in the magnitude of the cost of the drug treatment, the viral load, and immune effectors in the objective functional (3.1). Figure 3 shows the results for the hybrid on-off method. Figure 4 shows the simple STI and associated solutions for comparison purposes. We note that drugs are interrupted during treatment. Such interruptions can cause extremely high viral load and thus lead to an increase in infected cells, stimulating an immune response. Even when the drug is discontinued, because of a strong immune response in both strategies, the viral load remains low and the population of uninfected CD4+ T cells recovers from the effect of HIV. That is, the derived on-off control can move the model system to a "healthy" steady state in early infection. We observe that the drug is discontinued earlier in the hybrid on-off control than in the simple STI control.

We now provide an example of the movement between the model's two stable equilibria $\left(E Q_{1}\right.$ and $\left.E Q_{3}\right)$ as discussed in previous sections. Here we identify the control functions that move the state from an "unhealthy" equilibrium to a "healthy" one. We use the "unhealthy" stable equilibrium $\left(E Q_{1}\right)$ as the initial condition for model (2.1) for the time interval [0,990] (that is, $S(0)=164.197$, $I(0)=11.936, V(0)=63.628$, and $E(0)=0.024)$ to demonstrate that this 
equilibrium can be moved to a "healthy stable equilibrium through the hybrid on-off control. Figure 5 shows the hybrid on-off control and its associated solutions, and Figure 6 shows the simple STI control and its associated solutions. The virus and immune effector populations shift from an "unhealthy" equilibrium showing high viral load and low immune effector counts to a "healthy" equilibrium showing low viral load and high immune effector counts. Both the hybrid on-off control and the simple STI control suggest that the optimal use of STIs can boost the immune response and enhance subsequent control of viral load without requiring drugs. However, the use of the hybrid on-off control allows for the derivation of a more efficient scheme for leveraging the immune response to achieve similar outcomes. In particular, the drug is discontinued much earlier in the hybrid on-off control than in the simple STI control. In addition, the viral load remains low, and the population of uninfected CD4+ $\mathrm{T}$ cells recovers from HIV. Therefore, the hybrid on-off control proposes optimal therapeutic options that can move patients from an "unhealthy" stable equilibrium to a "healthy" one within a shorter treatment period.

\section{Conclusions}
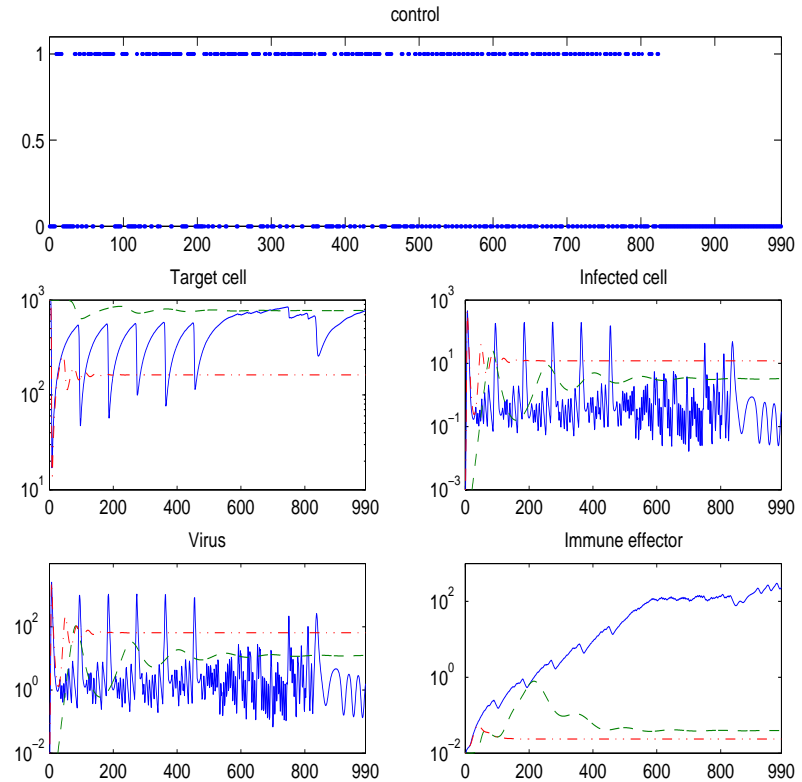

FIGURE 3. The hybrid on-off control and its solutions (solid line) with $P=1, Q=10^{-2}$, and $R=5 \times 10^{2}$; solutions with full-treatment (dashed line); and solutions with no-treatment (dashed and dotted line) for early infection 

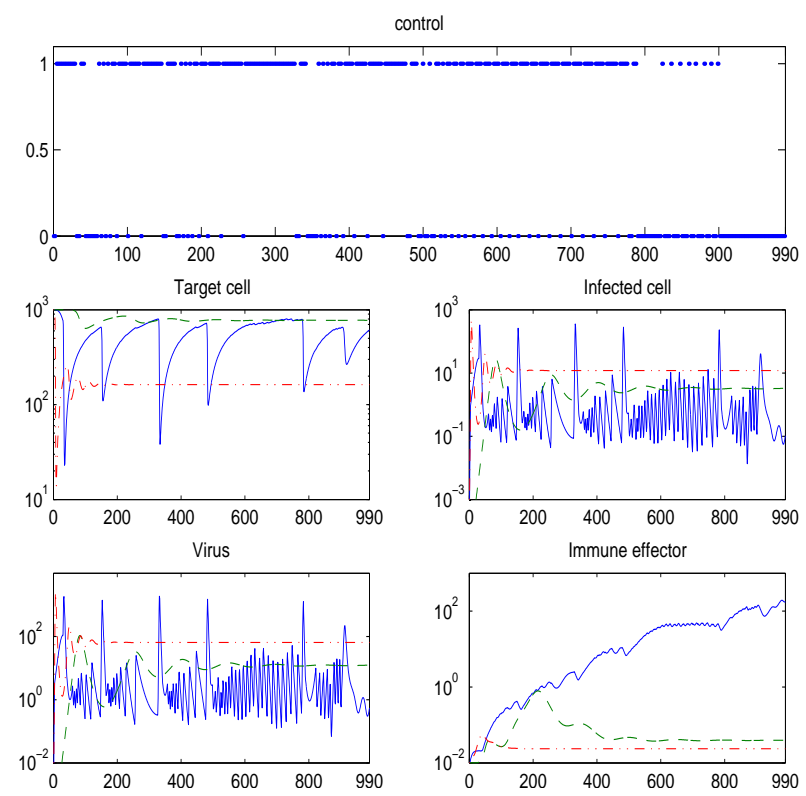

Figure 4. The simple STI control and its solutions (solid line) with $P=1, Q=10^{-2}$, and $R=5 \times 10^{2}$; solutions with full-treatment (dashed line); and solutions with no-treatment (dashed and dotted line) for early infection

In this paper we have considered how to design and synthesize efficient and practical therapy strategies for HIV. The mathematical model for HIV progression consists of compartments for target cells, infected cells, virus, and immune response, which exhibits multiple locally stable steady states. For the model considered we apply techniques and ideas from optimal control theory. The optimal control problem is formulated to achieve the goal of suppressing virus and boosting immune effector while being mindful of drug usage subject to a drug treatment as a control. The linear control problem yields continuous control that has the potential to suggest an optimal therapeutic option being able to minimize the viral load and to maximize the immune response. However, the resulting treatment regime is not practical in a clinical setting due to the continuous singular controls for some intervals in which the switching function is zero.

In this context, we propose a hybrid on-off control combining the linear optimal control and the direct search method, in particular, the simple structured treatment interruption (STI) introduced in $[1,2]$. As a result, we obtain a practical on-off therapy protocol that can move the model system to a healthy steady state in which the immune response is dominant in controlling 

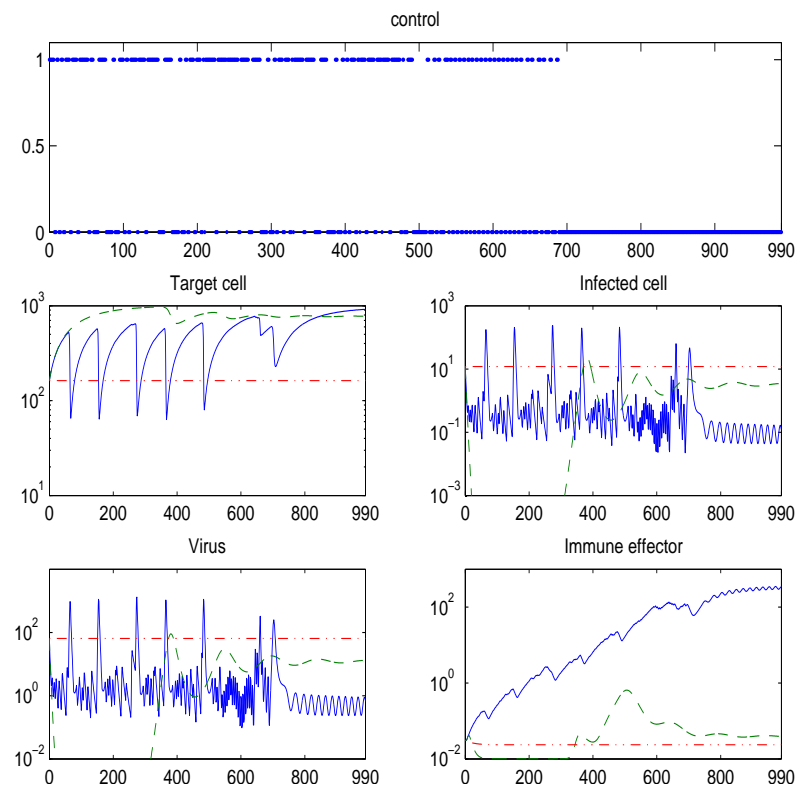

Figure 5. The hybrid on-off control and its solutions (solid line) with $P=1, Q=10^{-2}$, and $R=5 \times 10^{2}$; solutions with full-treatment (dashed line); and solutions with no-treatment (dashed and dotted line) in the unhealthy steady state

HIV. Moreover, numerical simulations show that hybrid on-off control attains smaller values of the objective functional than simple STI and allows for shorter treatment period. In summary, we conclude that hybrid on-off control function provides a more efficient strategy compared to simple STI, maintaining clinical benefits of simple STI control to lead to long-term control of HIV after the discontinuation of the therapy.

The proposed hybrid on-off control has a great potential to provide guidelines for HIV treatment strategies, but further research is necessary to be implemented in a clinical setting. While we demonstrated that viral load at low levels for long periods is maintained through hybrid on-off control, the actual results may vary from patient to patient. One important reason is that the model parameters are assumed to be fixed, but everybody has different values, in fact. In order to address this issue, more work is needed to verify the robustness of our algorithm. Another direction to advance is incorporating feedback control approach to reflect the patient's status not only at the initial stage but also at the follow-up visits. 

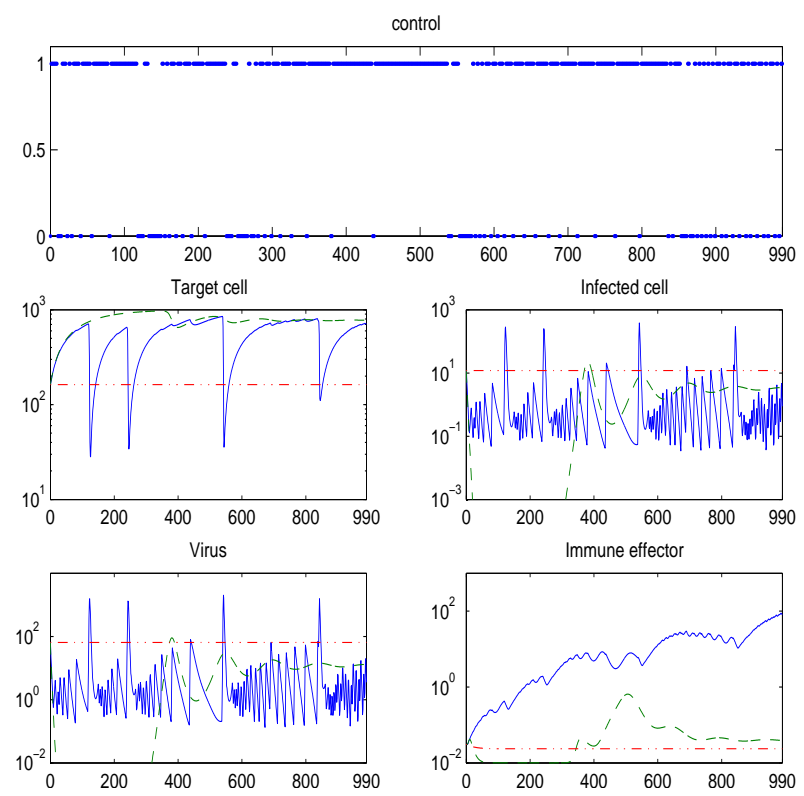

FIgURE 6 . The simple STI control and its solutions (solid line) with $P=1, Q=10^{-2}$, and $R=5 \times 10^{2}$; solutions with full-treatment (dashed line); and solutions with no-treatment (dashed and dotted line) in the unhealthy steady state

Acknowledgements. Authors would like to thank referees for their valuable comments to improve the paper. The work of Hee-Dae Kwon was supported in part by the NRF Grant funded by the Korean government (NRF2012R1A1A2005605) and by Inha University Research Grant. The work of Jeehyun Lee was supported by Basic Science Research Program through the NRF funded by the Ministry of Education(2013R1A1A2058848).

\section{References}

[1] B. M. Adams, H. T. Banks, M. Davidian, H. D. Kwon, H. T. Tran, S. N. Wynne, and E. S. Rosenberg, HIV dynamics: Modeling, data analysis, and optimal treatment protocols, J. Comput. Appl. Math. 184 (2005), no. 1, 10-49.

[2] B. M. Adams, H. T. Banks, H. D. Kwon, and H. T. Tran, Dynamic multidrug therapies for HIV: Optimal and STI control approaches, Math. Biosci. Eng. I (2004), no. 2, 223241.

[3] J. Alvarez-Ramirez, M. Meraz, and J. X. Velasco-Hernandez, Feedback control of the chemotherapy of HIV, Int. J. Bifur. Chaos 10 (2000), 2207-2219.

[4] H. T. Banks, H. D. Kwon, J. A. Toivanen, and H. T. Tran, A state-dependent Riccati equation-based estimator approach for HIV feedback control, Optimal Control Appl. Methods 27 (2006), no. 2, 93-121. 
[5] S. Bonhoeffer, M. Rembiszewski, G. M. Ortiz, and D. F. Nixon, Risks and benefits of structured antiretroviral drug therapy interruptions in HIV-1 infection, AIDS 14 (2000), 2313-2322.

[6] M. E. Brandt and G. Chen, Feedback control of a biodynamical model of HIV-1, IEEE Trans. on Biom. Engrg. 48 (2001), 754-759.

[7] D. S. Callaway and A. S. Perelson, HIV-1 infection and low steady state viral loads, Bull. Math. Biol. 64 (2001), 29-64.

[8] A. Carr and D. A. Cooper, Adverse effects of antiretroviral therapy, Lancet 356 (2000), 1423-1430.

[9] Y. H. Dai, L. Z. Liao, and D. Li, On restart procedures for the conjugate gradient method, Numer. Algorithms 35 (2004), no. 2-4, 249-260.

[10] K. R. Fister, S. Lenhart, and J. S. McNally, Optimizing chemotherapy in an HIV model, Electron. J. Differential Equation 1998 (1998), no. 32, 1-12.

[11] W. H. Fleming and R. W. Rishel, Deterministic and Stochastic Optimal Control, Springer-Verlag, New York, 1975.

[12] J. C. Gilbert and J. Nocedal, Global convergence properties of conjugate gradient methods for optimization, SIAM J. Optim. 2 (1992), no. 1, 21-42.

[13] B. Jülg and F. D. Goebel, Treatment interruption in HIV therapy: A SMART Strategy?, Infection 34 (2006), 186-288.

[14] D. Kirschner, S. Lenhart, and S. Serbin, Optimal control of the chemotherapy of HIV, J. Math. Bio. 35 (1997), no. 7, 775-792.

[15] L. S. Lasdon, S. K. Mitter, and A. D. Waren, The conjugate gradient method for optimal control problems, IEEE Trans. Automatic Control AC-12 (1967), no. 2, 132-138.

[16] J. Lisziewicz and F. Lori, Structured treatment interruptions in HIV/AIDS therapy, Microbes and Infection 4 (2002), 207-214.

[17] F. Lori, R. Maserati, et al., Structured treatment interruptions to control HIV-1 infection, The Lancet 354 (2000), 287-288.

[18] D. L. Lukes, Differential Equations: Classical to Controlled, Mathematics in Science and Engineering, Academic Press, New York, 1982.

[19] E. L. Murphy, A. C. Collier, L. A. Kalish, S. F. Assmann, M. F. Para, T. P. Flanigan, P. N. Kumar, L. Mintz, R. R. Wallach, and G. J. Nemo, Highly active antiretroviral therapy decreases mortality and morbidity in patients with advanced HIV disease, Ann. Intern. Med. 135 (2001), 17-26.

[20] G. C. Palacios, L. M. Sanchez, E. Briones, T. J. Ramirez, H. Castillo, L. G. Rivera, C. A. Vazquez, C. Rodriguez-Padilla, and M. Holodniy, Structured interruptions of highly active antiretroviral therapy in cycles of 4 weeks off/12 weeks on therapy in children having a chronically undetectable viral load cause progressively smaller viral rebounds, Int. J. Infect. Dis. 14 (2010), e34-e40.

[21] F. J. Palella Jr, K. M. Delaney, A. C. Moorman, M. O. Loveless, J. Fuhrer, G. A. Satten, D. J. Aschman, S. D. Holmberg, Declining morbidity and mortality among patients with advanced human immunodeficiency virus infection, HIV Putpatient Study Investigators, N. Engl. J. Med. 338 (1998), 853-860.

[22] L. S. Pontryagin, V. G. Boltyanskii, R. V. Gamkrelidze, and E. F. Mishchenko, The Mathematical Theory of Optimal Processes, Interscience Publishers John Wiley and Sons, Inc. 528, New York-London, 28, 1962.

[23] E. S. Rosenberg, M. Davidian, and H. T. Banks, Using mathematical modeling and control to develop structured treatment interruption strategies for HIV infection, Drug and Alcohol Dependence 88S (2007), S41-S51.

[24] Z. J. Shi and J. Guo, A new algorithm of nonlinear conjugate gradient method with strong convergence, Comput. Appl. Math. 27 (2008), no. 1, 93-106. 
[25] H. Shim, S. J. Han, C. C. Chung, S. Nam, and J. H. Seo, Optimal scheduling of drug treatment for HIV infection: Continuous dose control and receding horixon control, Int. J. Control Autom. Systems 1 (2003), 401-407.

[26] J. D. Siliciano, J. Kajdas, D. Finzi, T. C. Quinn, K. Chadwick, J. B. Margolick, C. Kovacs, S. J. Gange, and R. F. Siliciano, Long-term follow-up studies confirm the stability of the latent reservoir for HIV-1 in resting CD $4+T$ cells, Nat. Med. 9 (2003), 727-728.

[27] H. M. Speigel, et al., Changes in frequency of HIV-1 specific cytotoxic T cell precursors and circulating effectors after combination anti-retroviral therapy in children, J. Inf. Dis. 180 (1999), 359-368.

[28] S. Tang, Y. Xiao, N. Wang, and $\mathrm{H}$. Wu, Piecewise HIV virus dynamic model with CD4+ $T$ cell count-guided therapy: I, J. Theoret. Biol. 308 (2012), 123-134.

[29] L. M. Wein, S. A. Zenios, and M. A. Nowak, Dynamic multidrug therapies for HIV: A control theoretic approach, J. Theoret. Biol. 185 (1997), 15-29.

[30] D. Wodarz and M. A. Nowak, Specific therapy regimes could lead to long-term immunological control of HIV, Proceedings of the National Academy of Sciences 96 (1999), 4464-14469.

TAE SOO Jang

Department of Mathematics

INHA UNIVERSITY

INCHEON 402-751, KOREA

E-mail address: iamnumber2@inha.ac.kr

JUNGEUN KIM

Department of Computational Science and Engineering

YoNSEI UNIVERSITY

SeOul 120-749, Korea

E-mail address: jek.o.lantern@gmail.com

Hee-Dae Kwon

Department of Mathematics

INHA UNIVERSITY

INCHEON 402-751, KOREA

E-mail address: hdkwon@inha.ac.kr

JEEHYUN LEE

Department of Mathematics and

Department of Computational Science and Engineering

Yonsei University

SEOUL 120-749, Korea

E-mail address: ezhyun@yonsei.ac.kr 